\title{
ON KNOT INVARIANTS RELATED TO SOME STATISTICAL MECHANICAL MODELS
}

\author{
V. F. R. JONES \\ Dedicated to the memory of Henry Dye
}

\begin{abstract}
We use three different kinds of statistical mechanical models to construct link invariants. The vertex models emerge as the most general. Our treatment of them is essentially the same as Turaev's. Using the work of Goldschmidt we are able to define models whose invariants are homology invariants for branched covers. Thus the statistical mechanical framework embraces both the "classical" and the "new" link invariants.
\end{abstract}

0. Introduction. In this paper we shall discuss three types of statistical mechanical models-vertex models, Potts type models, and IRF models. In all cases we shall see that the models may be defined on a knot diagram (replacing the lattice of the model), and that a suitable variation on the partition function of the system is often a knot invariant, i.e. depends only on the knot as a three-dimensional entity and not on the chosen diagram.

The connection between knot theory and statistical mechanics was first established, indirectly, in [J1] where it was observed that the Temperley-Lieb algebra of the Potts and ice-type models (see [Ba] and [TL]) can be used to define a knot invariant using the theory of braids and a certain trace on the Temperley-Lieb algebra, discovered in the course of investigations into type $\mathrm{II}_{1}$ factors (see [J2]). (This invariant is a Laurent polynomial in $\sqrt{t}$ which we shall write $V_{L}(t)$, where $L$ is some oriented link.) But it was Kauffman who first began to understand this connection in a direct way with his "states model" for $V_{L}$, which freed the understanding of $V_{L}$ from the use of braids or inductive methods (as in $[\mathbf{F}+]$ ). Kauffman's model seems very special to $V_{L}$, but another approach to such explicit formulae was suggested by the braid formalism. The author succeeded in "unbraiding" a trace formula for a series of specializations of the two variable polynomial of $[\mathbf{F}+]$. The relevant braid group representations were discovered by Jimbo [Ji], Drinfeld [D], and Wenzl [W1]. This unbraiding was reported in a letter to Kauffman and we give the details of it in this paper. It was immediately generalized by Turaev [Tu] to embrace the Kauffman polynomial. We present our own version of Turaev's 
formalism, which has the interesting feature that it uses the angles formed at the crossings of a knot projection.

All the formulae referred to in the preceding paragraph are analogues of the partition function for a statistical mechanical system on a two-dimensional diagram of the link. Turaev's formulae correspond to a certain class of models known as "vertex models". We shall see that for Potts-type and IRF models it is possible to define non-trivial link invariants as analogues of the partition function. Vertex models turn out to be the most general, though in a perhaps artificial way.

The formalism is interesting for its generality. Using the work of Goldschmidt and the author in [GJ] we recover models which calculate the homology of 2-fold branched covers, and in view of [Go] it seems likely that all of the information in a Seifert matrix is accessible to our method. We are also able to situate Kauffman's model quite clearly as an intermediary between the Potts model and a vertex model (called "ice-type") via a piece of combinatorics that seems unique to the Potts model and the ice-type model.

Although the formalism is quite general and not tied to braid presentations or induction, it is still hampered by the need for a twodimensional projection (shadow) of a three-dimensional object. Our main reason for doing this work was as a step towards a useful and genuinely three-dimensional understanding of the invariants. So far we have not succeeded. The situation is the same as that of the poor prisoners in Plato's allegory of the cave [P1, pp. 253-254].

1. Vertex models. In statistical mechanics a vertex model is defined on a graph with vertices $V=\{v\}$ and edges $\mathscr{E}=\{e\}$. A finite set $\Theta$ (or more generally, a measure space) of "states per edge" is given and a state of the system is a function $\sigma: \mathscr{E} \rightarrow \Theta$. Let $\mathscr{H}$ be the set of all states. For each vertex $v$ there is an energy function $E_{v}: \mathscr{H} \rightarrow \mathbf{R}$ such that $E_{v}(\sigma)$ is determined by the restriction of $\sigma$ to the set of edges incident to $v$ (nearest neighbor interaction). The partition function of the vertex model is the sum $Z=\sum_{\sigma \in \mathscr{H}} \exp \left(-(1 / k T)\left(\sum_{v} E_{v}(\sigma)\right)\right)$. If the system is in interaction with its surroundings a term needs to be added to $\sum_{v \in V} E_{v}(\sigma)$ (see [Hi]). This should be the origin of the "angle" term in our formalism below. The Boltzmann weights are the terms $w_{v}(\sigma)=\exp \left(-(1 / k T) E_{v}(\sigma)\right)$ so that $Z=\sum_{\sigma \in \mathscr{H}} \prod_{v \in V} w_{v}(\sigma)$. The $w_{v}(\sigma)$ are in general functions of certain variables.

We shall be interested in four-valent graphs only and the vertices will be divided into two classes, "+" and "-", so that $w_{v}(\sigma)=$ $w_{ \pm}(a, b \mid x, y)$ where $\{a, b, x, y\}$ are the elements of $\Theta$ assigned by $\sigma$ to 
the edges incident to $v$, ordered according to some convention. Thus we make the following formal definition (which is intended for knot theory, the equations (1.2)-(1.5) are too restrictive for statistical mechanics).

Definition 1.1. A vertex model is a finite set $\Theta$ together with two functions $w_{ \pm}(a, b \mid x, y)(\lambda)=w_{ \pm}(a, b \mid x, y)(\lambda, h)$ where $a, b, x, y \in \Theta$, $\lambda \in(0, \pi)$ (and we are deliberately vague about the values of $w$; they should at least lie in a commutative ring), a "random variable" $f: \Theta \rightarrow$ $\mathbf{R}, f(a)=f_{a}$, and a constant $\hbar=h / 2 \pi$ such that

$$
\begin{gathered}
\sum_{b, y} w_{+}(a, b \mid y, x)(\lambda) w_{-}(z, y \mid b, c)(\lambda) \exp \left((\lambda-\pi) \hbar\left(f_{b}+f_{y}\right)\right) \\
=\delta(a, c) \delta(x, z) \exp \left((\lambda-\pi) \hbar\left(f_{a}+f_{z}\right)\right)
\end{gathered}
$$

$$
\begin{aligned}
& \sum_{b, y, s} w_{+}(a, b \mid x, y)(\lambda) w_{+}(b, c \mid r, s)(\lambda+\mu) w_{+}(y, z \mid s, t)(\mu) \\
& \quad=\sum_{b, y, s} w_{+}(x, y \mid r, s)(\mu) w_{+}(a, b \mid s, t)(\lambda+\mu) w_{+}(b, c \mid y, z)(\lambda) .
\end{aligned}
$$

Comments on Definition 1.1. We let $V$ be a vector space with basis $\Theta$ and define linear maps $R_{ \pm}(\lambda) \in \operatorname{End}(V \otimes V), \check{R}_{ \pm}(\lambda) \in \operatorname{End}(V \otimes V)$ and $L(\delta) \in \operatorname{End}(V)$ by

$$
\begin{aligned}
R_{ \pm}(\lambda)(a \otimes x) & =\sum_{b, y} w(a, b \mid x, y) b \otimes y, \\
\check{R}_{ \pm}(\lambda)(a \otimes x) & =\sum_{b, y} w(a, b \mid x, y) y \otimes b, \\
L(\delta) a & =\exp \left(\delta \hbar f_{a}\right) a .
\end{aligned}
$$

Also let $P \in \operatorname{End}(V \otimes V)$ be given by $P(a \otimes b)=b \otimes a$. Then note: $\check{R}_{ \pm}(\lambda)=P R_{ \pm}(\lambda)$. 
Equation (1.2) shows that $w_{ \pm}(a, b \mid x, y)(\lambda)$ may be extended to all $\lambda \in \mathbf{R}$ and that $w_{ \pm}(a, b \mid x, y)(0)$ determines $w_{ \pm}(a, b \mid x, y)(\lambda)$. Provided the image of exp contains no zero divisors, it follows that $w_{ \pm}(a, b \mid x, y)(\lambda)=0$ unless $f_{a}+f_{x}=f_{b}+f_{y}$. Thus our framework is no more general than Turaev's [Tu]. We may rewrite (1.2) as

$$
\begin{aligned}
R_{ \pm}(\lambda+\delta) & =(L(-\delta) \otimes 1) R_{ \pm}(\lambda)(L(-\delta) \otimes 1)^{-1} \\
& =(1 \otimes L(\delta)) R_{ \pm}(\lambda)(1 \otimes L(\delta))^{-1}
\end{aligned}
$$

so that in particular $\check{R}_{ \pm}(\lambda)$ commutes with $L(\delta) \otimes L(\delta)$ and $\check{R}_{ \pm}(\lambda)=$ $(L(\lambda) \otimes 1) \check{R}_{ \pm}(0)(1 \otimes L(-\lambda))$. We suppose that $(1.2)$ holds for the rest of the discussion.

Equation (1.3) is the same as

$$
\check{R}_{-}(0) \check{R}_{+}(0)=\mathrm{id} .
$$

Thus the values of $w_{-}(a, b \mid x, y)(\lambda)$ are determined by those of $w_{+}(a, b \mid x, y)(\lambda)$ and + and - can be interchanged in (1.2).

Equation (1.4) may be recast as follows. For $A \in \operatorname{End}(V \otimes V)$ let $A^{t_{1}}$ and $A^{t_{2}}$ be (transpose $\left.\otimes \mathrm{id}\right)(A)$ and (id $\otimes$ transpose) $(A)$ respectively. Then (1.4) is the same as

$$
\left[P\left(R_{+}(\pi)\right)^{t_{1}}\right]\left[P\left(R_{-}(\pi)\right)^{t_{2}}\right]=\text { id } .
$$

Equation (1.5) is the well-known Yang Baxter equation, to be found often in [Ba]. Using an obvious notation for the operations of elements of $\operatorname{End}(V \otimes V)$ on $V \otimes V \otimes V$ we can rewrite (1.5), with $R=R_{+}$, as

$$
R_{12}(\lambda) R_{23}(\lambda+\mu) R_{13}(\mu)=R_{13}(\mu) R_{23}(\lambda+\mu) R_{12}(\lambda) .
$$

Because we are supposing (1.2), this is equivalent to the braid relation with $\check{R}=\check{R}(0)$

$$
\check{R}_{12} \check{R}_{23} \check{R}_{12}=\check{R}_{23} \check{R}_{12} \check{R}_{23} \text {. }
$$

As we noted earlier, the conditions (1.2), (1.3), (1.4) are quite strong and the physically interesting solutions of (1.5) in [Ba] do not satisfy them.

Now suppose that we are given a generic planar projection of a smooth oriented link in $\mathbf{R}^{3}$, so that the only singularities are double points and at these the intersections are transversal. Then the link $L$ 


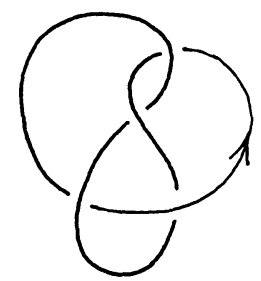

Figure 1.9

Diagram of an oriented link

may be adequately represented by the planar projection together with crossing data as shown in Figure 1.9.

DEFINITION 1.10. Given a vertex model $\nu=\left(\Theta, w_{ \pm}, f, \hbar\right)$ and an oriented link diagram $L$ we define the partition function as

$$
Z_{L}^{\nu}=\sum_{\text {states } \sigma}\left(\prod_{\substack{c \\ \text { cossings } \\ \text { of } L}} w_{ \pm}(a, b \mid x, y)(\lambda)\right) \exp \left(\hbar \int_{L} f_{\sigma} d \Theta\right)
$$

(an empty product is equal to 1) where a state $\sigma$ is a function from the edges of the planar graph subjacent to $L$ to $\Theta$. So a state defines a configuration around each crossing represented by Fig. 1.11 which also establishes the convention as to the order in which $a, b, x, y$ appear as arguments of $w_{ \pm}$
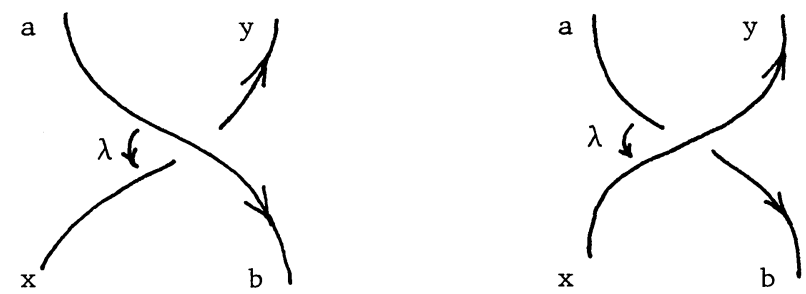

FIGURE 1.11

We take $\lambda$ to be the "ingoing" angle measured in radians, in the sense of Fig. 1.11. Obviously $0<\theta<\pi$.

Finally, $f_{\sigma}$ is the locally constant function on $L$ defined as being $f_{\sigma(e)}$ along any edge $e$, and $d \theta$ is the "change of angle" or curvature 1 -form on $L$, defined as the pull-back to $L$ of the angle form on the circle via the mapping $L \rightarrow S^{1}$ given by a unit tangent vector pointing in the direction of the orientation of $L$. This ends Definition 1.10. 
Regular isotopy is the equivalence relation on link diagrams (generated by planar isotopy and the (oriented) Reidemeister moves of types II and III (see [BZ]).

THEOREM 1.12. Given a vertex model $\nu$ and two regularly isotopic link diagrams $L_{1}$ and $L_{2}, Z_{L_{1}}^{\nu}=Z_{L_{2}}^{\nu}$.

Proof. Equation (1.2) implies that each term in $Z_{L}^{\nu}$ is unchanged by changing the angles made at the crossings. It follows fairly easily that each term in $Z_{L}^{\nu}$ is invariant under planar isotopy. Once this is established, (1.3), (1.4) and (1.5) are clearly sufficient conditions for $Z_{L}^{\nu}$ to be invariant under all type II Reidemeister moves and a special configuration of type III move. Invariance under the other oriented type III moves follows by manipulating $\left(1.2^{\prime}\right)$ and $\left(1.5^{\prime \prime}\right)$, and a simple topological argument in one case. We refer to [Tu] for details.

One is most interested in isotopy of links in $\mathbf{R}^{3}$ which is expressed by invariance under all the Reidemeister moves, but, as emphasized by Kauffman, any regular isotopy invariant provided one pays attention to the Tait number Tait $(L)$ (=number of crossings, counted according to their sign) and the rotation number $\operatorname{rot}(L)$. But all the examples we shall give behave in a particularly simple way under the type I Reidemeister moves which we now formalize.

Definition 1.13. A vertex model $\nu=(\Theta, w, f, \hbar)$ has the type I property if

$$
\left\{\begin{array}{c}
\sum_{a} w_{ \pm}(a, b \mid x, a)(\lambda) e^{\hbar(2 \pi-\lambda) f_{a}}=\delta(x, b) e^{-\lambda \hbar f_{b}} \\
=\sum_{a} w_{ \pm}(b, a \mid a, x)(\lambda) e^{-\hbar(2 \pi-\lambda) f_{a}} \\
=\delta(x, b) e^{+\lambda \hbar f_{b}} .
\end{array}\right.
$$

COROLlary 1.15. If $\nu$ is a vertex model with the type I property then $Z_{L}^{\nu}$ depends only on $L$ up to isotopy.

(Often a model will not have the type I property but can be changed into one with it simply by multiplying $w_{+}$by a constant factor and $w_{-}$by its inverse.)

Let us write $\operatorname{tr}_{1}$ and $\operatorname{tr}_{2}$ for the partial traces from $\operatorname{End}(V \otimes V) \rightarrow$ End $V$ (e.g. if $A(a \otimes x)=\sum A(a, b \mid x, y)(b \otimes y)$, then $\operatorname{tr}_{1}(A)(x)=$ $\left.\sum_{y, a} A(a, a \mid x, y) y\right)$.

Equation (1.14) is equivalent to

$$
\operatorname{tr}_{1}\left(\check{R}_{ \pm}(2 \pi)\right)=L(-2 \pi), \quad \operatorname{tr}_{2}\left(\check{R}_{ \pm}(2 \pi)\right)=L(2 \pi)
$$


or

$\left(1.14^{\prime \prime}\right) \operatorname{tr}_{1}\left((L(2 \pi) \otimes 1) \check{R}_{ \pm}(0)\right)=\mathrm{id}, \quad \operatorname{tr}_{2}\left(\check{R}_{ \pm}(0)(1 \otimes L(-2 \pi))\right)=\mathrm{id}$

We see that, in the notation of Turaev, $R_{i j}^{k l}=w(i, l \mid j, k)(0)$ and $\mu_{i}=$ $e^{h f_{i}}, \hbar=h / 2 \pi$, when the set $\Theta$ is the set $\{1,2, \ldots, n\}$.

Examples of vertex models.

EXAMPLE 1.16. We begin with the example reported in a letter to Kauffman. For $n=1,2, \ldots$, let $P_{n}$ be the vertex model defined by $\Theta=\{-n,-n+2, \ldots, n-2, n\}, f_{a}=a$ and

$$
\begin{gathered}
w_{+}(a, b \mid x, y)(\lambda, h) \\
= \begin{cases}0 & \text { if }\{a, x\} \neq\{y, b\} \text { or } a<b, \\
\exp \left(\frac{h}{2}\right) & \text { if } a=b=x=y, \\
1 & \text { if } a=b, x=y, a \neq x, \\
2 \sinh \left(\frac{h}{2}\right) \exp (\lambda \hbar(b-a)) & \text { if } a>b, a=y, b=x,\end{cases} \\
\quad w_{-}(a, b \mid x, y)(\lambda, h)=w_{+}(x, y \mid a, b)(\lambda,-h) .
\end{gathered}
$$

Then (1.2)-(1.5) are satisfied. It is easy to show that if $\sigma$ is given, the term $T=\left(\prod_{\nu} \exp (\hbar(b-a))\right) \exp \left(\hbar \int_{L} \sigma d \theta\right)$ may be calculated as follows: replace any crossing with $a>b, a=y, b=x$ by the "smoothed" picture as in Fig. 1.17 (similarly for negative crossings).
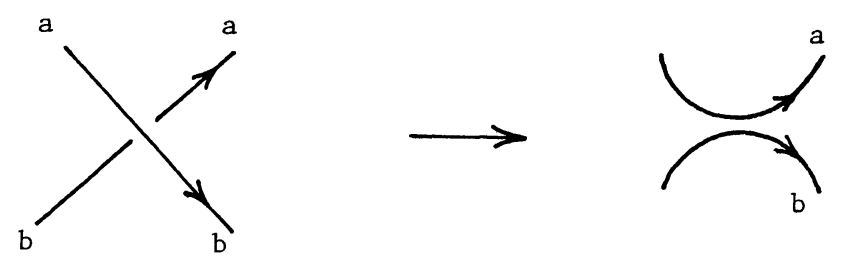

FIGURE 1.17

In the resulting diagram $L^{\prime}, \sigma$ is constant along connected components and $T=\exp \left(h \sum_{a=-n}^{+n} a \operatorname{rot}\left(L_{a}^{\prime}\right)\right)$ where $L_{a}^{\prime}$ is the subdiagram of $L^{\prime}$ consisting of those connected components for which $\sigma=a$.

The vertex models $P_{n}$ can be multiplied by a factor so that they have the type I property. Let $P_{L}(n, h)$ denote the partition function of this normalized model. One may show

(a) that $P_{L}(n, h)$ is a Laurent polynomial in $e^{h / 2}$ divisible by $P_{\text {unknot }}(n, h)$ 
(b) that if $L_{+}, L_{-}$and $L_{0}$ are link diagrams identical except in the neighborhood of one crossing, where they are as in Fig. 1.18 , then

$$
\begin{aligned}
& \exp \left((n+1) \frac{h}{2}\right) P_{L_{+}}(n, h)-\exp \left(-\frac{(n+1) h}{2}\right) P_{L_{-}}(n, h) \\
& \quad=\sinh \left(\frac{h}{2}\right) P_{L_{0}}(n, h)
\end{aligned}
$$

Thus as $n$ varies, the $P_{L}(n, h)$ define a family of specializations of the two-variable invariant of $[\mathbf{F}+]$, sufficient to determine it (and thus prove its existence).
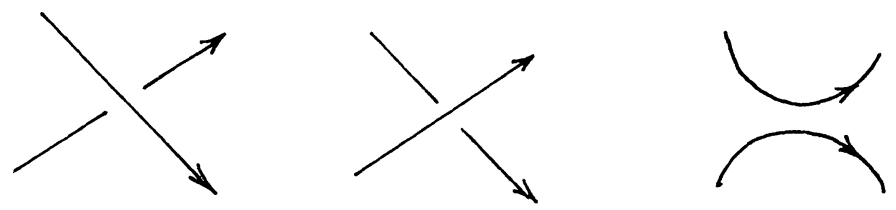

FIGURE 1.18

EXAMPLE 1.19. Turaev's examples in [Tu] defining the Kauffman polynomial satisfy our equations with the obvious choice of $w_{ \pm}, f$, $q=e^{h / 2}$.

EXAMPLE 1.20. The quantum group formalism of [Ji], [Dr] suggests that there is a vertex model invariant associated with any finite dimensional representation of any complex simple Lie algebra. Indeed, Example 1.18 corresponds to $s l_{n}$ in its $n$ dimensional identity representation and Example 1.19 embraces the $B_{n}, C_{n}$ and $D_{n}$ series in their fundamental representations. In support of this conjecture we give another example, corresponding to the $N$-dimensional irreducible representation of $s l_{2}$. The matrix $R(0)$ can be deduced from [Dr] and [Ji2]. These examples have apparently also been discovered using braids by Akutsu and Wadati [AW] and Wenzl [W2] although it is difficult to be absolutely sure, as only the first three cases are given in [AW] and only an existence result occurs in [W2] (which also gives many other invariants in support of the conjecture). Our models are as follows. For each $N=2,3,4, \ldots$, let $X_{2 n}$ be the vertex model with

$$
\Theta=\left\{-\frac{N-1}{2},-\frac{N-1}{2}+1, \ldots, \frac{N-3}{2}, \frac{N-1}{2}\right\},
$$


$f_{a}=a$ and $w(a, b \mid x, y)(\lambda, h)$ defined by

$$
\begin{aligned}
& w_{+}(a, b \mid x, y)(\lambda, h) \\
& =\left\{\begin{array}{c}
\exp \left\{\frac{h}{2}\left[a x+b y+\frac{k(k-1)}{2}+\frac{\lambda}{\pi}(a-b)\right]\right\} \Gamma_{x, k}^{N}(h) \\
\text { where } k=a-b \text { if } a+x=b+y \text { and } a \geq b, \\
0 \quad \text { otherwise }
\end{array}\right.
\end{aligned}
$$

and $w_{-}(a, b \mid x, y)(\lambda, h)=w_{+}(x, y \mid a, b)(\lambda,-h)$, where

$$
\Gamma_{x, k}^{N}(h)=2^{k} \prod_{j=0}^{k-1} \frac{\sinh \left(\frac{h}{2}\left(x+\frac{N+1}{2}+j\right)\right) \sinh \left(\frac{h}{2}\left(x-\frac{N-1}{2}+j\right)\right)}{\sinh \left(-\frac{h}{2}(1+j)\right)}
$$

(empty products are one).

I would like to thank M. Rosso for some help with $w_{-}$. One sees immediately that (20) and (22) of [Tu] are satisfied, with $\mu_{a}=\exp (h a)$, $\nu_{a}=-\exp (h a / 2)$ and $a^{\prime}=-a$, so that (17) of [Tu], which is a version of our (1.4), is satisfied. This also proves that the invariants of this example are, up to powers of $e^{h}$, invariants of unoriented links. Equation (1.2) is obvious and equation (1.3) follows from direction computation. Equation (1.5) follows from [Dr] and [Ji2].

Using the notation of [An], the identity $\sum_{\alpha=0}^{\beta}\left(q^{-\beta}\right)_{\alpha}(z)_{\alpha} q^{\alpha} /(q)_{\alpha}=$ $z^{\beta}$ is easily proven by induction (or follows from the proof of (3.3.12) of [An] as pointed out to the author by Andrews). It shows that $w_{ \pm}$ may be multiplied by powers of $e^{h}$ so that the normalized vertex model has the type I property. The resulting link invariant is a Laurent polynomial in $e^{h / 2}$. The case $N=2$ is the same as the case $n=1$ of Example 1.18, namely, the polynomial $V_{L}$. W. Baxter has written a program for calculating these invariants using braids. The method is effective for $N \leq 5$ for braids on three strings and for $N \leq 4$ for braids on five strings.

EXAMPLE 1.21. This example was discovered by A. Lipson [Li]. It has the feature that the Boltzmann weights are $\lambda$-independent. We show how to deduce these weights from invariant theory, as pointed out by R. Howe.

If $E$ is $n$-dimensional Euclidean space with inner product $\langle$,$\rangle , there$ is a privileged element $e$ of $\operatorname{End}(E \otimes E)$ which is orthogonal projection onto the one-dimensional subspace spanned by $\sum v_{i} \otimes v_{i}$, where $\left\{v_{i}\right\}$ is an orthonormal basis. It follows immediately that $e_{1} e_{2} e_{1}=\left(1 / n^{2}\right) e_{1}$, $e_{2} e_{1} e_{2}=\left(1 / n^{2}\right) e_{2}$ in $\operatorname{End}(E \otimes E \otimes E)$ where $e_{1}=e_{12}, e_{2}=e_{23}$ in the 
notation of $\left(1.5^{\prime}\right)$. By the calculations of [Jo4], if we put $\check{R}_{ \pm}(\lambda)=$ $\left(t^{ \pm 1}+1\right) e-1$ where $4 \cosh ^{2}(h / 2)=n^{2}, t=e^{h}$, then the coefficients of the matrix $\check{R}$ give Boltzmann weights satisfying (1.3) and (1.5). Explicitly we define the model with $\Theta=\{1,2, \ldots, n\}, f_{a}=0$ and

$$
\begin{aligned}
& w_{+}(a, b \mid x, y)(\lambda, h) \\
& = \begin{cases}0 & \text { if } a \notin\{x, y\} \text { or } x \notin\{a, b\}, \\
\exp (-h / 4) & \text { if } a=y, x=b, a \neq b, \\
\exp (h / 4) & \text { if } a=x, y=b, a \neq b, \\
2 \cosh (h / 4) & \text { if } a=x=y=b,\end{cases} \\
& w_{-}(a, b \mid x, y)(\lambda, h)=w_{+}(a, b \mid x, y)(\lambda,-h) .
\end{aligned}
$$

By inspection the assignment of the Boltzmann weights at a crossing is independent of the directions of the arrows. Thus (1.4) is the same as (1.3). The type I property is also satisfied after normalization. This example obviously calculates $V_{L}\left(e^{h}\right)$.

EXAMPLE 1.22. Another unoriented example with no angle dependence due to Lipson is defined in [Li], [LM]. It is trivial on knots and determined in general by linking numbers of sublinks with their complements.

We see that even vertex models with no angle dependence can give highly non-trivial information. It would be nice to know if such models are also sufficient to determine the invariants of Examples 1.18, 1.19 and 1.20 .

We add the final comment that (1.2)-(1.5) do not mix $\{a, b\}$ and $\{x, y\}$ so one should be able to define invariants that treat the different components of a link differently.

2. Spin models, the Potts model, IRF models. We use the terminology "spin model" to denote a statistical mechanical model, such as the Potts model, defined on a graph with vertices $\{v\}$ and edges $\{e\}$. The vertices may be in a (finite) set $\Theta$ of "spins" and a state is a function $\sigma:\{v\} \rightarrow \Theta$. The model is defined by an energy function $E: \Theta \times \Theta \rightarrow \mathbf{R}$ so that the energy of an edge joining vertices $v_{1}$ and $v_{2}$ is $E\left(\sigma\left(v_{1}\right), \sigma\left(v_{2}\right)\right)=E(a, b)$. As before write $w(a, b)=\exp (-\beta E(a, b))$. The partition function is then

$$
\sum_{\sigma} \exp \left(-\beta \sum_{e} E(a, b)\right)=\sum_{\sigma} \prod_{e} w(a, b) .
$$

Although it is possible to develop a more precise theory for oriented links, all our examples will be unoriented, so we now outline a theory, 
analogous to that of $\S 1$, which allows us to construct invariants of unoriented links as partition functions of spin models.

DEFINITION 2.1. A spin model $S=\left\{\Theta, w_{ \pm}\right\}$will be a set of $n$ "spins" $\Theta$ and functions $w_{ \pm}(a, b), a, b \in \Theta$ such that (for all $a, b, c \in \Theta$ )

$$
\begin{gathered}
w_{ \pm}(a, b)=w_{ \pm}(b, a), \\
w_{+}(a, b) w_{-}(a, b)=1, \\
\sum_{x \in \Theta} w_{-}(a, x) w_{+}(x, c)=n \delta(a, c),
\end{gathered}
$$

$$
\sum_{x \in \Theta} w_{+}(a, x) w_{+}(b, x) w_{-}(c, x)=\sqrt{n} w_{+}(a, b) w_{-}(b, c) w_{-}(c, a) .
$$

(Equation (2.5) is known as the "star-triangle relation" and occurs essentially in [On].)

Definition 2.6. Let $S=\left(\Theta, w_{ \pm}\right)$be a spin model and $L$ be an $u n$ oriented link diagram. We define the partition function $Z_{L}^{S}$ as follows.

First we shade the regions of $L$ black and white so that adjacent regions have different colours and the unbounded region is white as in Figure 2.7:

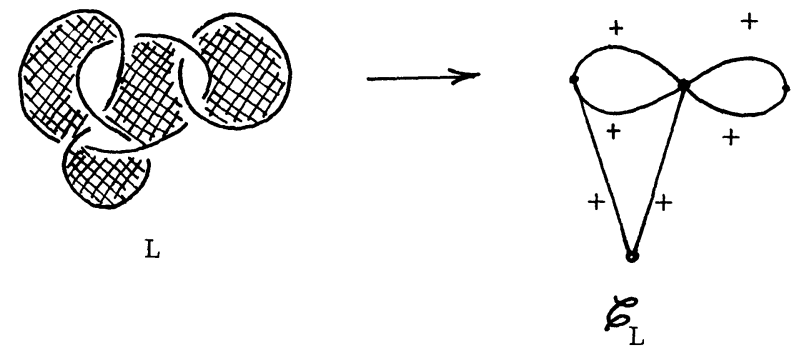

FIGURE 2.7

We then form the planar graph $\mathscr{C}_{L}$, with signed edges, with $V$ vertices which are the black regions of $L$ and whose edges are the crossings of $L$. One may distinguish two types of crossings of $L$, equivalently, edges of $\mathscr{C}_{L}$ according to a convention established by Fig. 2.9.

A state $\sigma$ will be a function from the vertices of $\mathscr{H}_{L}$ to $\Theta$, and given a state $\sigma$ and an edge $e$ between vertices $v_{1}$ and $v_{2}$ with sign + or -, we will write $w_{ \pm}(a, b)$ for $w_{ \pm}\left(\sigma\left(v_{1}\right), \sigma\left(v_{2}\right)\right)$. Then we set

$$
Z_{L}^{S}=\left(\frac{1}{\sqrt{n}}\right)^{V-1} \sum_{\text {states edges }} \prod_{ \pm}(a, b),
$$

where as usual an empty product is one. This ends Definition 2.6. 
THEOREM 2.8. If $S$ is a spin model and $L_{1}$ and $L_{2}$ are unoriented connected link diagrams then $Z_{L_{1}}^{S}=Z_{L_{2}}^{S}$ if $L_{1}$ and $L_{2}$ are regularly isotopic.

Proof. Clearly $Z^{S}$ does not change under planar isotopy. Invariance under type II Reidemeister moves is guaranteed by (2.3) and (2.4) corresponding to the type II moves

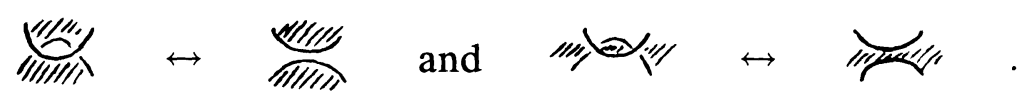

Note that in the second case the number of shaded regions changes which necessitates a normalization of the form $(1 / \sqrt{n})^{V}$. The number of shaded regions may be assumed to change by two in this move since the $L_{1}$ and $L_{2}$ are connected.

There are two kinds of type III moves to be considered as shown in Fig. 2.9.

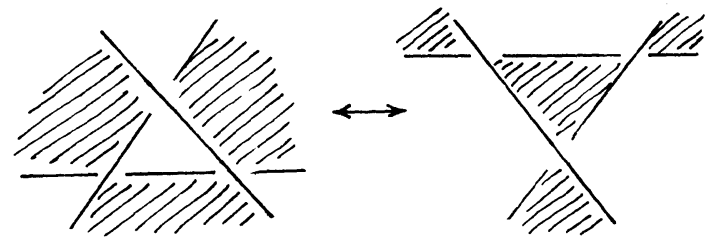

or
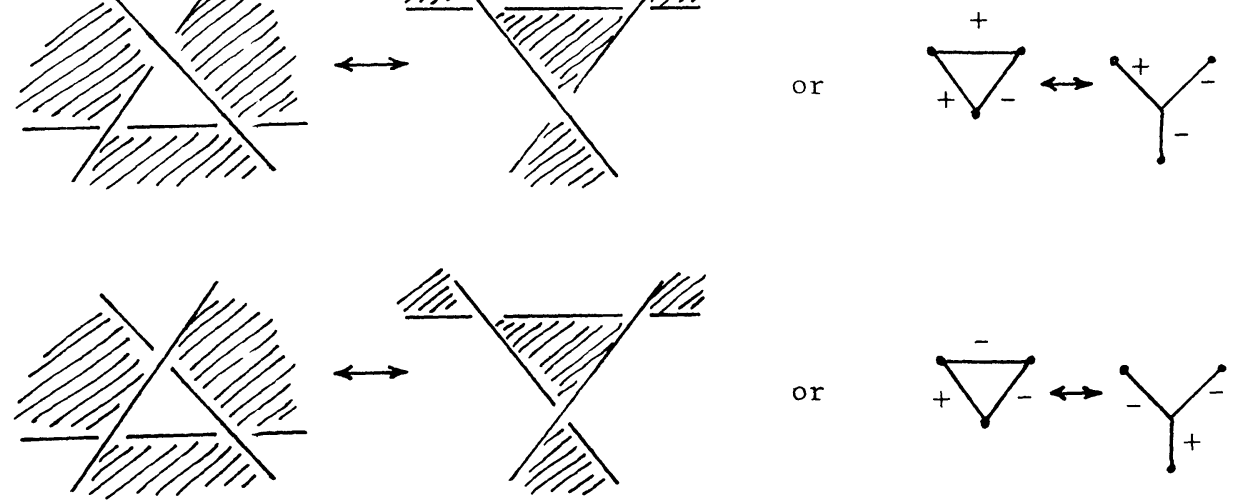

or

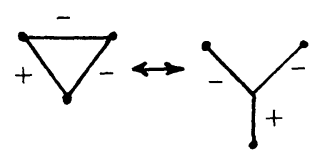

FIGURE 2.9

Invariance under the first kind is assured by (2.5). Invariance under the second kind is given by the following:

$$
\begin{aligned}
& \sum_{x \in \Theta} w_{+}(a, x) w_{-}(b, x) w_{-}(c, x) \\
& =\sqrt{n} w_{+}(a, b) w_{-}(b, c) w_{+}(c, a) .
\end{aligned}
$$

Now consider an $n$-dimensional vector space $V$ with basis $\Theta$ and on $V \otimes V$ define the operators $X$ and $\Delta$ by $X(a \otimes b)=\sum_{x} w_{+}(a, b) x \otimes b$ and $\Delta(a \otimes b)=w_{-}(a, b) a \otimes b$. Then (2.5) is the same as

$$
X \Delta X=\sqrt{n} \Delta X \Delta
$$


and by $(2.2)-(2.4),(2.10)$ is the same as

$$
X \Delta X^{-1}=\frac{1}{\sqrt{n}} \Delta^{-1} X \Delta .
$$

Perhaps surprisingly, all spin models have the type I property of (1.14) in the following sense. (Note that type I Reidemeister moves are positive or negative according to the following convention:

$$
+1 \rightarrow \lambda,-1 \rightarrow \text { 尺 .) }
$$

Proposition 2.11. Let $(S, \Theta)$ be a spin model. Then there is a constant $A$, called the modulus of $S$ such that if $L^{\prime}$ is obtained from $L$ by adding a loop via a \pm 1 type I Reidemeister move then $Z_{L^{\prime}}=A^{\mp 1} Z_{L}$.

Proof. Putting $b=c$ in (2.5) and using (2.3) we have $\sum_{x \in \Theta} w_{+}(a, x)$ $=\sqrt{n} w_{-}(b, b)$ for all $a, b$. If we set

$$
A=w_{-}(b, b)=(1 / \sqrt{n}) \sum_{x \in \Theta} w_{+}(a, x)
$$

then a careful analysis of the shading possibilities proves the result.

Thus if $\vec{L}$ is an oriented link with associated unoriented link $L$, and $S$ is a spin system of modulus $A$, we may define the quantity

$$
Z_{\vec{L}}^{S}=A^{\operatorname{Tait}(\vec{L})} Z_{L}^{S}
$$

Proposition 2.13. $Z_{\vec{L}}^{S}$ is an invariant of oriented links.

Proof. Just note that both possible orientations give the sign +1 to the crossing involved in a positive type I Reidemeister move.

Note that the reasoning of Theorem 2.8 shows that we would have obtained a link invariant $\check{Z}_{L}^{S}$ for the spin model $S$ by agreeing to colour the unbounded region black. The next result shows that nothing new happens. Note that the result is not entirely trivial as the two graphs corresponding to the different colourings may look very different. 
Proposition 2.14 (Duality). If $S$ is a spin model and $L$ is an oriented link projection, then $Z_{L}^{S}=\check{Z}_{L}^{S}$.

Proof. The sequence of Reidemeister moves of Fig. 2.15 converts $L$ into $L^{\prime}$ such that the white-unbounded colouring of $L^{\prime}$ is the same as the black-unbounded colouring of $L$.

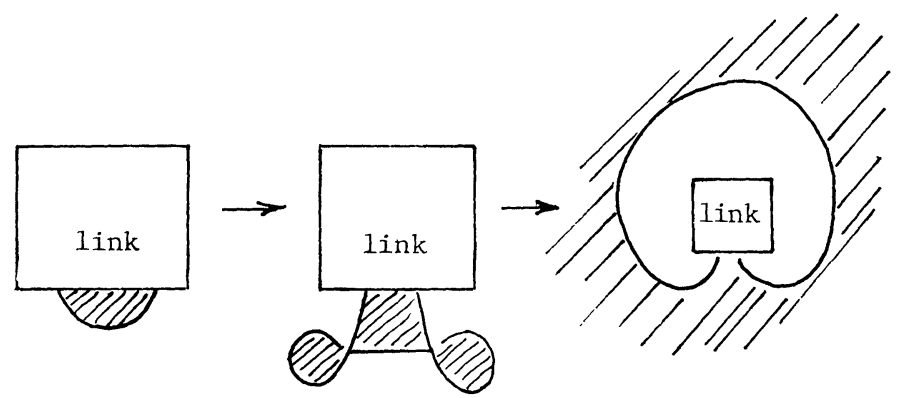

FIGURE 2.15

Note that equations (2.2)-(2.5) impose many restrictions on $w_{ \pm}(a, b)$. The following is quite useful.

Proposition 2.16. For each $z$ let $k_{z}$ be the number of ordered pairs $(a, b)$ for which $w_{+}(a, b)=z$. Then $k_{z}$ is a multiple of $n\left(\right.$ and $\sum_{z} k_{z}=$ $\left.n^{2}\right)$.

Proof. By $\left(2.13^{\prime}\right)$ the matrices $\Delta$ and $1 / \sqrt{n} X$ are conjugate, but $\Delta$ is diagonal with eigenvalues $w_{-}(a, b)$ and $X$ is of the form $\pi \otimes$ id on $V \otimes V$ so that the multiplicities of all of its eigenvalues are multiples of $n$.

EXAMPles. With Proposition 2.16 it is easy to find all solutions (2.2)-(2.5) when $n=2$ or 3 . We leave it to the reader to check that one obtains the invariants $V_{L}(i), V_{L}\left(e^{i \pi / 3}\right)$ and nothing more. When $n=4$ one also obtains the invariant of (1.22) and some others of no great significance. We shall be especially interested in the following two examples for both of which the set $\Theta$ can be given the structure of a group so that $w_{+}(a, b)$ depends only on $a b^{-1}$.

EXAmple 2.17 (The Potts model). In statistical mechanics the Potts model is the spin model for which the Boltzmann weights depend only on whether the two atoms are in the same state or not. Correct choice of the parameters leads, for each $n$, to the following choice of $w_{ \pm}$(by 
(2.3) it suffices to give $\left.w_{+}\right)$:

$$
w_{+}(a, b)= \begin{cases}1 & \text { if } a=b, \\ -t^{-1} & \text { otherwise, }\end{cases}
$$

where $2+t+t^{-1}=n$. It is easy to check that these Boltzmann weights satisfy (2.2)-(2.6). One may also check directly that the invariant of unoriented links is an unoriented version of $V_{L}(t)$.

EXAMPLE 2.18 (Metaplectic invariants) [GJ]. If the set $\Theta$ is a group $G$, we may impose the condition that $w_{+}(a, b)$ depend only on $a b^{-1}$, say $w_{+}(a, b)=f\left(a b^{-1}\right)$. Then (2.2), (2.4) and (2.5) become

$$
\left\{\begin{array}{l}
\text { (a) } f(g)=f\left(g^{-1}\right), \\
\text { (b) } \frac{1}{|G|} \sum_{h \in G} f(h)\left[f\left(h^{-1} g\right)\right]^{-1}=\delta_{g, 1}, \\
\text { (c) } f(x y) f(x)^{-1} f(y)^{-1} \\
\quad=\frac{1}{\sqrt{|G|}} \sum_{g \in G} f\left(y^{-1} g\right) f(x g) f(g)^{-1} .
\end{array}\right.
$$

If $G=\mathbf{Z} / n \mathbf{Z}$ we see that the Boltzmann weights of Example 2.17 satisfy (2.19). Another example, with $G=\mathbf{Z} / n \mathbf{Z}, n$ odd, which coincides with Example 2.17 when $n=3$ but differs for $n>3$, was discovered in [GJ]. The function $f(a)$ is a Gaussian: $f(a)=K e^{\pi i a^{2} / n}$ where $K^{-2}=\sum_{a \in \mathbf{Z} / n \mathbf{Z}} e^{2 \pi i a^{2} / n}$. The knot invariant was shown in [GJ] to be determined by a Seifert matrix and to be essentially given by the rank of the homology of the twofold branched cover of $S^{3}$, branched over the knot, with $\bmod n$ coefficients.

A lot of "classical" knot invariants were obtained in [GJ]. It seems likely that they may all be accessible to the partition function method using slightly more elaborate spin models. Thus the formalism is able to embrace both the "classical" (Seifert matrix) and "new" $\left(V_{L}, P_{L}\right.$, Kauffman polynomial) invariants as well.

I would like to thank D. Goldschmidt and T. Ziman for many stimulating discussions about spin models.

The spin models present many approachable problems. In general they seem more susceptible to brute force calculations than other models because the whole data is just an $n \times n$ matrix. It would be quite feasible to determine all models for $n=5,6$. It would also be nice to discover a machine, analogous to the quantum group machine, for creating solutions to $(2.2)-(2.5)$.

IRF Models. IRF (interaction round a face) models are similar to spin models in that a state is defined by a function $\sigma$ : vertices of a lattice $\rightarrow \Theta$. They differ in that the energy of a state is the sum of 
the energies of each face, the energy of a given face being determined by the configuration of states on the vertices of its boundary. Thus if the lattice is a square lattice in two dimensions one is given a function $w(a, b, c, d), a, b, c, d \in \Theta$, and the partition function for a finite rectangular sublattice will be $\sum_{\text {states }} \prod_{\text {faces }} w(a, b, c, d)$ where $a, b, c$, $d$ are taken in some fixed order around each face. IRF models as such do not generalize conveniently to an arbitrary graph, but if $L$ is an oriented link projection, its underlying graph is 4-valent so all faces of the planar dual are quadrilaterals and we may proceed in a manner analogous to spin models.

Definition 2.19. An IRF model $I=\{\Theta, w\}$ will be a set on $n$ "spins" $\Theta$ and functions $w_{ \pm}(a, b, c, d)$ with $a, b, c, d \in \Theta$ such that (for all $a, b, c, d, e, f \in \Theta$ )

$$
\begin{aligned}
& \sum_{x \in \Theta} w_{ \pm}(a, b, a, x)=1, \\
& \sum_{x} w_{+}(a, b, x, d) w_{-}(x, b, e, d)=\delta_{a, e}, \\
& \sum_{x} w_{+}(d, a, b, x) w_{-}(b, e, d, x)=\delta_{a, e}, \\
& \sum_{x} w_{+}(a, b, x, f) w_{+}(b, c, d, x) w_{+}(x, d, e, f) \\
& \quad=\sum_{x} w_{+}(b, c, x, a) w_{+}(a, x, e, f) w_{+}(x, c, d, e) .
\end{aligned}
$$

Although we shall not construct any IRF models not derived from spin models, we define the link invariants associated with them.

Definition 2.24. Let $I=\left\{\Theta, w_{ \pm}\right\}$be an IRF model and $L$ an oriented link projection. We define the partition function as

$$
Z_{L}^{I}=\sum_{\text {states }}\left(\prod_{\substack{\text { faces } \\ \text { of } \mathscr{C}}} w_{ \pm}(a, b, c, d)\right)
$$

where $\mathscr{H}$ is the planar dual of the graph subjacent to $L$, with signed faces which are + or - according to the sign of the unique crossing of $L$ which they contain. A state $\sigma$ is a function from the vertices of $\mathscr{H}$ to $\Theta$. For a given face in the product we choose $w_{ \pm}(a, b, c, d)$ according to the sign of the face and the configuration of spins at its four corners, ordered according to Fig. 2.25.

This ends Definition 2.24. 


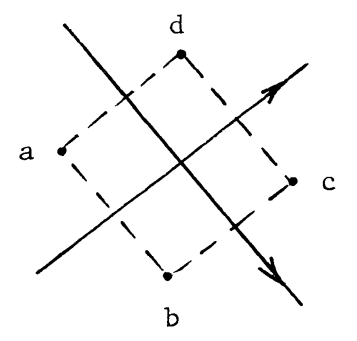

FIGURE 2.25

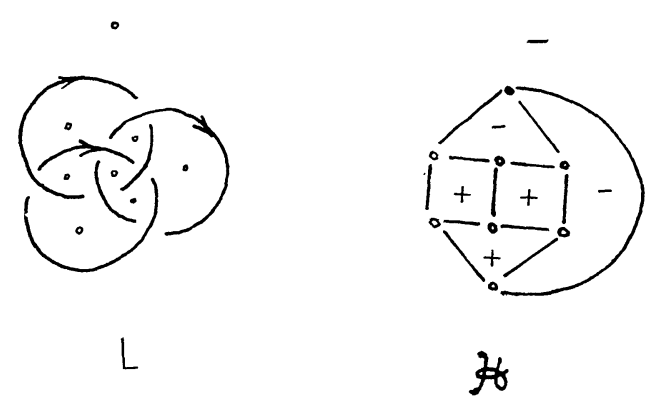

FIGURE 2.26

An example of the passage from $L$ to $\mathscr{H}$ is given in Fig. 2.26. The proof of the next result is trivial.

THEOREM 2.27. If I is an IRF model and $L_{1}$ and $L_{2}$ isotopic link models then $Z_{L_{1}}^{I}=Z_{L_{2}}^{I}$.

IRF models have also been considered from the braid point of view by Akutsu and Wadati [AW2]. Note that we could have dropped (2.20) if we had only been interested in regular isotopy.

3. Braids and transfer matrices. The role of braid group representations in knot theory is closely analogous to that of transfer matrices in statistical mechanics. Any oriented link can be represented as a closed braid, which can thus be thought of as a particularly ordered way to represent a link. To each of the partition function invariants presented above there is a braid group representation such that the trace of the braid is equal to the partition function. (In the case of vertex models with non-trivial angle dependence the trace must be suitably weighted.) In statistical mechanics the system is typically on a lattice, so is already well ordered. The technique of transfer matrices associates to each row of the lattice a (large) matrix, the trace of some power of which is the partition function for a (finite) square lattice 
with periodic boundary conditions. The row-to-row transfer matrix can often be written as a product of matrices corresponding to the atoms in that row, the matrix entries being the Boltzmann weights. These transfer matrices associated to the atoms correspond to the matrices representing the usual generators of the braid group.

The plat closure of a braid (see [Bi]) can sometimes be used. This corresponds to using different boundary conditions (non-periodic) on the square lattice (see [Jo3]).

We now outline the braid group representations in the three cases. It will be apparent how to construct the transfer matrices corresponding to atoms in the statistical mechanics concept. They can be found in [Ba]. In all cases the Yang Baxter equation or star triangle equation is a sufficient condition which ensures commuting row-to-row transfer matrices for different values of the "spectral parameter". We have been unable to use the spectral parameter in the spin and IRF cases. For the vertex models it becomes our "angle".

3.1. Vertex models. With notation as in $\S 1$, and $V$ as usual, a vector space with basis $\Theta$, we represent the braid group $B_{k}$ on $\otimes^{k} V$ using the $\check{R}(0)$ matrix which acts on $V \otimes V$ by

$$
\check{R}(0)(a \otimes x)=\sum_{b, y} w_{+}(a, b \mid x, y) y \otimes b .
$$

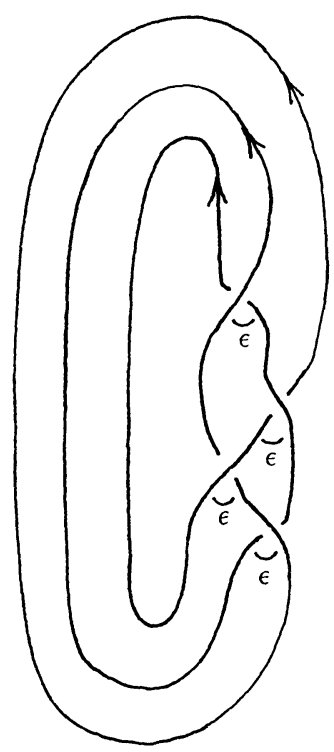

FIGURE 3.2 
We define $\check{R}_{i}$ on $\otimes^{k} V$ by

$$
\begin{aligned}
& \check{R}_{i}\left(\left(v_{1} \otimes \cdots \otimes v_{i-1}\right) \otimes v_{i} \otimes v_{i+1} \otimes\left(v_{i+1} \otimes \cdots \otimes v_{k}\right)\right) \\
& \quad=\left(v_{1} \otimes \cdots \otimes v_{i-1}\right) \otimes \check{R}(0)\left(v_{i} \otimes v_{i+1}\right) \otimes\left(v_{i+1} \otimes \cdots \otimes v_{k}\right) .
\end{aligned}
$$

Then $\check{R}_{i}^{-1}$ is obtained by applying the same prescription to $\check{R}^{-1}(0)$. Thus we get a representation of $B_{k}$ by sending $\sigma_{i}$ to $\check{R}_{i}$. We define the linear functional $\phi$ on $\operatorname{End}\left(\otimes^{k} V\right)$ by $\phi(A)=\operatorname{trace}(D A)$ where $D=L(2 \pi) \otimes L(2 \pi) \otimes \cdots \otimes L(2 \pi)$. If $\alpha$ is a braid in $B_{k}$ with closure $\hat{\alpha}$ then we may arrange the braid picture of $\hat{\alpha}$ to look as in Fig. 3.2.

The angle $\varepsilon$ may be made arbitrarily small so that the only angle contribution to the partition function is "around the back" of the braid. We see that in the limit, $\varepsilon \rightarrow 0$, we have $\phi(\alpha)=Z_{\hat{\alpha}}$ (see 5.2 of [Tu]). This is in fact how the partition function approach to $P_{L}$ was discovered, in an attempt to reverse the procedure just outlined.

3.3. Spin models. Given the Boltzmann weights $w_{ \pm}(a, b)$ and a vector space $V$ with basis $\Theta$ we consider three ways to form elements of $\operatorname{End}(V \otimes V)$, already used in Theorem 2.11. Let

$$
\begin{aligned}
\Delta(a \otimes b) & =w_{-}(a, b) a \otimes b, \\
X_{1}(a \otimes b) & =\frac{1}{\sqrt{n}} \sum_{x} w_{+}(a, x) v_{x} \otimes v_{b} \quad \text { and } \\
X_{2}(a \otimes b) & =\frac{1}{\sqrt{n}} \sum_{x} w_{+}(b, x) v_{a} \otimes v_{x} .
\end{aligned}
$$

Obviously, $X_{1}$ and $X_{2}$ commute and (2.5) implies $\Delta X_{1} \Delta=X_{1} \Delta X_{1}$ and $\Delta X_{2} \Delta=X_{2} \Delta X_{2}$. Moreover $\Delta^{-1}, X_{1}^{-1}$ and $X_{2}^{-1}$ exist by (2.3) and (2.4). Thus we may define a representation of $B_{2 k}$ on $\bigotimes_{i=1}^{k} V$ by sending $\sigma_{2 i-1}$ to $\Delta_{i}$, which is $\Delta$ on the $i$ th tensor factor, tensored with the identity on the others, and $\sigma_{2 i}$ to $X_{i}$ where

$$
\begin{gathered}
X_{i}\left(u_{1} \otimes u_{2} \otimes u_{3}\right)=u_{1} \otimes X\left(u_{2}\right) \otimes u_{3} \text { where } \\
u_{1} \in \bigotimes_{j=1}^{i-1} V, \quad u_{2} \in V, \quad u_{3} \in \bigotimes_{j=k+1}^{k} V \quad \text { and } \quad X_{1}=X \otimes \text { id } .
\end{gathered}
$$

We leave it to the reader to contemplate Fig. 3.4 and see that, for a braid $\alpha \in B_{2 k}$, the trace of the matrix representing $\alpha$ on $\otimes^{k} V$ is the same (up to normalization) as the partition function invariant of $\hat{\alpha}$. Notice how the matrix $\Delta$, using $w_{-}$, corresponds to horizontal bonds, whereas $X$, corresponding to vertical ones, uses $w_{+}$. Note also that duality occurs in the braid picture as invariance of the trace under a shift "half a step" to the right. 


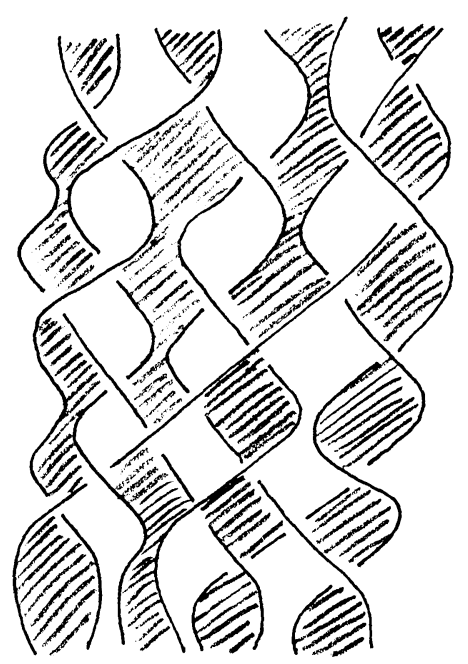

FIGURE 3.4

3.5. IRF models. Given the Boltzmann weights $w_{ \pm}(a, b, c, d)$ of an IRF model and $V$ with basis $\Theta$, we define an operator $R$ on $V \otimes V \otimes V$ by

$$
R(b \otimes a \otimes d)=\sum_{c} w_{+}(a, b, c, d) b \otimes c \otimes d .
$$

Then by (2.21) $R^{-1}$ exists and is given by the same formula with $w_{+}$ replaced by $w_{-}$. If we define $R_{1}$ and $R_{2}$ on $V \otimes V \otimes V \otimes V$ by $R_{1}=R \otimes$ id and $R_{2}$ by id $\otimes R$ then (2.23) is the same as $R_{1} R_{2} R_{1}=R_{2} R_{1} R_{2}$. So we may define representations of $B_{k}$ on $\otimes^{k+1} V$ in what should by now be an obvious manner, shifting $R$ to the right by one for each succeeding generator. We leave it to the reader to contemplate Fig. 3.4, without the shading and with $\times$ 's in all the regions, to establish that the trace of a braid $\alpha$ in this representation is the same as the partition function invariant of $\hat{\alpha}$.

We end $\S 3$ by pointing out a puzzling feature common to all the models, related to Markov's theorem [Ma]. It is that, from the braid point of view, all that is required for a link invariant is invariance under the Markov moves (see [J1] or [Tu]). This is guaranteed by the equations for the model expressing invariance under the types I and III Reidemeister moves. The existence of an inverse matrix is also required, invoking one kind of type II equation ((1.3) or (2.21)) but not the other. Thus one may find $R$ matrices defining invariants via the braid representation picture, but not defining models as above. Indeed here is an example. Let $X$ and $Y$ be defined on the vector space $V$ with basis $v_{1}, v_{2}, \ldots, v_{n}$ by $X v_{k}=\omega^{k} v_{k}, Y v_{k}=v_{(k+1) \bmod n}(\omega=$ $\left.e^{2 \pi i / n}\right)$. Then defining $\check{R} \in \operatorname{End}(V \otimes V)$ by $\check{R}=\sum_{k \in \mathbf{Z} / n \mathbf{Z}} \omega^{k^{2}} X^{k} \otimes Y^{k}$ 
it is easy to check that all the conditions for a vertex model except (1.4) are satisfied (with $f_{a}=0$ ). In fact, the trace of the braid group representation calculates the invariants of [GJ] outlined in (2.18).

On the other hand, if we adopt the regular isotopy point of view we may forget about type I Reidemeister moves and one could imagine models for which the braid/trace picture did not behave in any simple way under the type II Markov moves. The situation on these points seems unclear at this juncture.

4. Equivalences. The author would like to thank H. Au-Yang and J. Perk for pointing out (in statistical mechanics) the existence for every spin model of an equivalent IRF model, and for every IRF model of an equivalent vertex model. These equivalences persist in the knot theory context but are more subtle because of positive and negative crossings. It is also true that, in statistical mechanics, to every vertex model on a square lattice there is an equivalent IRF model (see [PW]) but we have been unable to make such an equivalence in the knot theory version. For these reasons we state our equivalences as formal results.

Proposition 4.1. Let $S=\left\{\Theta, w_{ \pm}\right\}$be a proper spin model. Then there is an IRF model $I=\left\{\Theta, \tilde{w}_{ \pm}\right\}$such that $Z_{L}^{I}=\left(Z_{L}^{S}\right)^{2}$ for all oriented connected link diagrams $L$, where we forget the orientation of $L$ for $Z_{L}^{S}$.

Proof. Let $\Theta$ have $n$ elements. Set

$$
\tilde{w}_{ \pm}(a, b, c, d)=(1 / \sqrt{n}) w_{ \pm}(a, c) w_{\mp}(b, d) .
$$

It is easy to check $(2.20) \rightarrow(2.23)$. Note that for a given link diagram $L$, the set of vertices $\mathscr{C}$ of the graph $\mathscr{H}$ is the union of the set of vertices for $\mathscr{C}$ and its dual $\check{\mathscr{C}}$. The faces of $\mathscr{H}$ are quadrilaterals whose diagonals are the edges of $\mathscr{C}$ and $\check{\mathscr{C}}$. So to each state of $\mathscr{H}$ there are unique states of $\mathscr{C}$ and $\check{\mathscr{C}}$. Thus by (2.14) we have

$$
\begin{aligned}
\left(Z_{L}^{S}\right)^{2} & =\check{Z}_{L}^{S} Z_{L}^{S} \\
& =(\sqrt{n})^{\#(\text { faces of } L)+2} \sum_{\substack{\text { states } \\
\text { of } \mathscr{C}}}\left(\prod_{\begin{array}{c}
\text { edges } \\
\text { of } \mathscr{C}
\end{array}} w_{\varepsilon}(a, c)\right)\left(\prod_{\begin{array}{c}
\text { edges } \\
\text { of } \mathscr{C}
\end{array}} w_{\mathcal{E}^{\prime}}(b, d)\right)
\end{aligned}
$$

where $\varepsilon, \varepsilon^{\prime}$ are + and -. Pairing up the products according to the faces of $\mathscr{H}$, the proof is completed by observing that the choice of $\varepsilon$ 

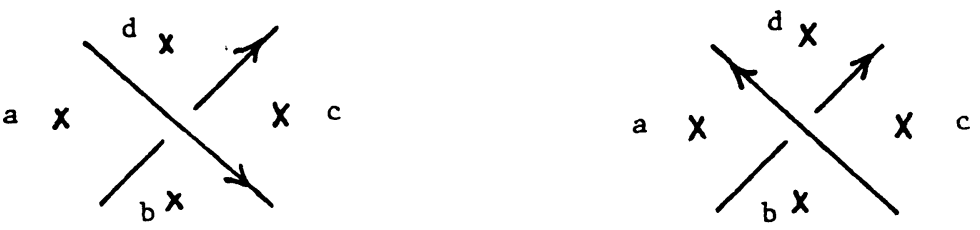

FIGURE 4.2

according to Fig. 2.10 corresponds to our definition of $\tilde{w}_{ \pm}(a, b, c, d)$, regardless of the orientation at a crossing. See Figures 4.2 and 2.25.

Note also that since $L$ is connected, \#(faces of $L)=\#($ faces of $\mathscr{H})+2$.

Proposition 4.3. Let $I=\left\{\Theta, w_{ \pm}\right\}$be an IRF model. Then there exists a vertex model $v=\left\{\Theta \times \Theta, \tilde{w}_{ \pm}, f\right\}$ (with $f \equiv 0$ ) for which $n Z_{L}^{I}=Z_{L}^{v}$ for any oriented link. Moreover $v$ has the type I property.

Proof. Define

$$
\begin{aligned}
& \tilde{w}_{ \pm}\left((d, a),(b, c) \mid\left(a^{\prime}, b^{\prime}\right)\left(c^{\prime}, d^{\prime}\right)\right) \\
& \quad= \begin{cases}w_{ \pm}(a, b, c, d) & \text { if }(a, b, c, d)=\left(a^{\prime}, b^{\prime}, c^{\prime}, d^{\prime}\right), \\
0 & \text { otherwise. }\end{cases}
\end{aligned}
$$

It is easily checked that this defines a vertex model and

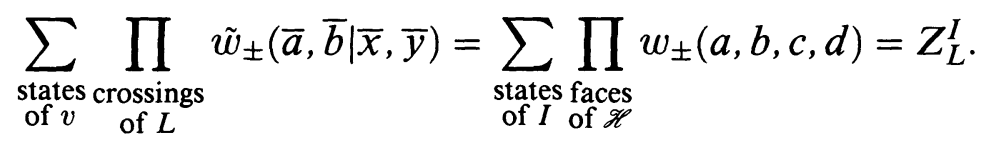

Thus up to a choice of square root, vertex models are more general than spin models and IRF models. We would add, however, that this should not be taken as a reason to ignore the spin and IRF models as the above equivalences are a little trivial and the natural context for the spin model is not the vertex model. For instance, the number of sites per edge is the square of the number of spins per site, which immediately makes the vertex model approach rather cumbersome.

For particular models there may be non-trivial equivalences between spin and vertex models. For instance, the vertex model defining $V_{L}$ $(n=1$ in Example 1.16) is equivalent to the collection of all Potts models (Example 2.17) by what is known to physicists as TemperleyLieb equivalence. This was discovered in [TL] using the transfer matrix picture but a direct proof for arbitrary planar graphs is given in [Ba]. The idea is to represent the two states per edge of the vertex 
model by arrows and given a state of the model, to eliminate all crossings according to the arrow configuration at the crossing. A clever counting argument shows that one obtains the partition function for the Potts model. The idea of eliminating the crossing according to the configuration around a vertex gives precisely the states model of Kauffman for $V_{L}$ (see [K1], [K2]). Thus the Kauffman states model is an intermediate step in the proof of Temperley-Lieb equivalence.

\section{REFERENCES}

[An] G. Andrews, The Theory of Partitions, Addison-Wesley, 1976.

[AW1] Y. Akutsu and M. Wadati, Exactly solvable models and new link polynomials, I: $N$-state vertex models, preprint 1987.

[Ba] R. Baxter, Exactly Solved Models in Statistical Mechanics, Academic Press, London, 1982.

[Bi] J. Birman, Braids, links and mapping class groups, Ann. Math. Studies, 82 (1974).

[BZ] G. Burde and H. Zieschang, Knots, DeGruyter, 1985.

[Dr] V. Drinfeld, Quantum groups in Proceedings of the International Congress of Mathematicians, Berkeley 1986, vol. 1, 798-820.

[F+] P. Freyd, D. Yetter, J. Hoste, W. Lickorish, K. Millet, and A. Ocneanu, A new polynomial invariant of knots and links, Bull. Amer. Math. Soc., 12 (1985), 183-312.

[GJ] D. Goldschmidt and V. Jones, Metaplectic link invariants, Univ. of California, Berkeley preprint (1987).

[Go] D. Goldschmidt, Classical link invariants via the Burau representation, Univ. of California, Berkeley preprint (1987).

[Hi] T. Hill, Statistical Mechanics, Dover Publications, Inc., 1956.

[Jil] M. Jimbo, A q-analogue of $U(\mathrm{gl}(n+1))$, Hecke algebra, and the Yang-Baxter equation, Letters in Math. Phys., (1986), 247-252.

[Ji2] _ A q-difference analogue of $U(\mathscr{H})$ and the Yang-Baxter equation, Letters in Math. Phys., 10 (1985), 63-69.

[Jol] V. Jones, A polynomial invariant for knots via von Neumann algebras, Bull. Amer. Math. Soc., 12 (1985), 103-111.

[Jo2] — Index for subfactors, Invent. Math., 72 (1983), 1-25.

[Jo3] _ Hecke algebra representations of braid groups and link polynomials, Ann. Math., 126 (1987), 335-388.

[Jo4] - Braid groups, Hecke algebras and type $\mathrm{II}_{1}$ factors, from Geometric Methods in Operator Algebras, Longman Sci. \& Tech., 1986, 242-273.

[K1] L. Kauffman, An invariant of regular isotopy, preprint.

[K2] State models and the Jones polynomial, Topology, 26 (1987), 395407.

[LM] W. B. R. Lickorish and K. Millet, An evaluation of the F polynomial of a link, preprint.

[Li] A. S. Lipson, Some more states models for link invariants, to appear in Pacific J. Math.

[Ma] A. A. Markov, Uber die freie Aquivalenz geschlossener Zopfe, Recevil Mathematique Moscou, 1 (1935), 73-78. 
[On] L. Onsager, Crystal statistics I. A two dimensional model with an orderdisorder transition, Phys. Rev., 65 (1944), 117-149.

[P1] Plato, The Republic (transl. B. Jowett), Prometheus Books, 367 B.C.

[PW] J. Perk and F. Wu, Graphical approach to the non-intersecting string model, Physica, 138A (1986), 100-124.

[TL] H. N. V. Temperley and E. Lieb, Relations between the "percolation" and "colouring" problem and other graph-theoretical problems associated with regular planar lattices: some exact results for the "percolation" problem, Proc. Roy. Soc. London, 322 (1971), 251-280.

[Tu] V. Turaev, The Yang-Baxter equation and invariants of links, LOMI preprint (Leningrad, 1987).

[W1] H. Wenzl, private communication.

[W2] Braid group representations and the quantum Yang-Baxter equation, (to appear).

Received April 15, 1988.

UNIVERSITY OF CALIFORNIA

BERKELEY, CA 94720 